\title{
AN ULTRASONICALLY POWERED ACTIVE STENT FOR ENDOVASCULAR DISEASES
}

\author{
Sayemul Islam ${ }^{l}$ and Albert Kim ${ }^{1 *}$
}

${ }^{1}$ Department of Electrical Engineering, Temple University, Philadelphia, Pennsylvania, USA

\section{ABSTRACT}

Endovascular aneurysm repair (EVAR) is a preferred treatment for patients with abdominal aortic aneurysm, especially for people who are not a good candidate for open surgery. However, the failure of endograft device can lead to post-EVAR sequela. It is imperative to develop an active stent that monitors the endovascular flow. This paper presents an ultrasonically powered active stent platform with a wireless sensing system. The active stent, which is an ultrasonic receiver is made of $110 \mu \mathrm{m}$-thick polyvinylidene fluoride (PVDF). The stent incorporates surface machined patterns that induce negative Poisson's ratio and power (i.e., vibration) enhancement. A cantilever-type flow sensor (cut from the PVDF) modulates the frequency of a surface integrated RF transmitter for wireless monitoring of blood flow. The flow sensor showed an average sensitivity of $0.11 \mathrm{mV} / \mathrm{mL} / \mathrm{min}$ when the RF transmitter could send the flow information 1 meter away to the receiver wirelessly with the frequency range of $81-88 \mathrm{MHz}$.

\section{INTRODUCTION}

Endovascular aneurysm repair (EVAR) is a minimally invasive treatment for abdominal aortic aneurysm patients by implanting a graft. It has been an exceedingly common technique, practiced since the first graft implantation in 1991 [1]. A bare-metal stent is inserted through a small incision and is positioned at the aneurysm site with an aid of image-guided system. The stent is then expanded within the artery to restore blood flow. This approach offers reduced postsurgical trauma, time under general anesthesia, length of hospital stays, and lowering mortality rates [2]. However, there are EVAR complications (e.g., arterial thrombosis, clot embolization into aortic side-branches, and pseudoaneurysm formation) due to device failure, procedural issues, and endoleaks which requires immediate surgical intervention [3]. Periodic monitoring of abdominal aortic blood flow after EVAR could predict such ischemic complications. In this paper, we report an ultrasonically powered active stent platform that incorporates a blood flow sensor and an RF transmitter to potentially minimize EVAR complications. Figure 1(a) shows a schematic view, and Figure 1(b) shows a block diagram of the active stent. The active stent consists of a surface machined and rolled PVDF sheet, a cantilever-type flow sensor, and surface integrated electronics (a rectifier, an amplifier, and LC tank modulated RF transmitter).

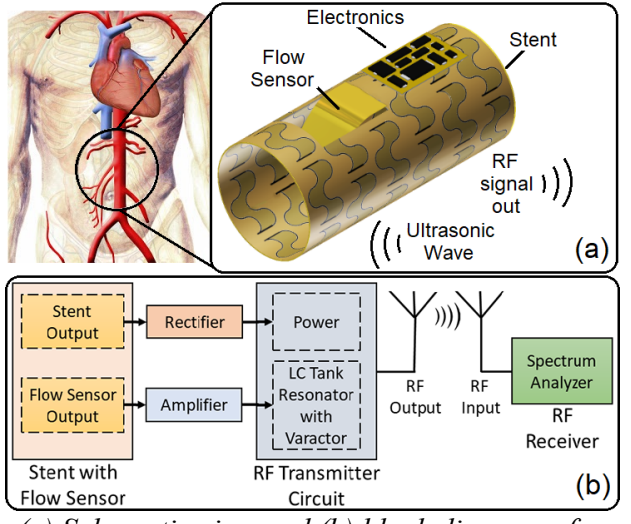

Figure 1: (a) Schematic view and (b) block diagram of an ultrasonically powered active stent with wireless flow sensor.

\section{MATERIALS AND METHODS}

As ultrasound is applied from outside the body, the stent with piezoelectric characteristics converts the mechanical energy to electrical power to energize the embedded sensor and electronics. The stent was designed with a surface pattern which has three important roles: 1) allowing shrinkage and expansion like traditional stents (negative Poisson's ratio), 2) providing anchoring, and 3) increasing power output by enabling vibration amplification [4].

For the flow sensor, a cantilever is cut from the main stent surface and bent towards the direction of blood flow. The flow induces deflection of the cantilever, which generates an electrical potential proportional to blood flow rate [5]. The electrical potential is then amplified (Gain $=37 \mathrm{~dB}$ ) and fed to a voltage-controlled varactor which is a part of the LC tank of the RF transmitter. The $7.5 \mathrm{~mm} \times 4 \mathrm{~mm}$ cantilever generated $53 \mathrm{mV}$ to $131 \mathrm{mV}$ for the flow rate range of $30 \mathrm{~mL} / \mathrm{min}$ to $980 \mathrm{~mL} / \mathrm{min}$, which is within the normal range of blood flow rate [6]. Both power and amplified sensor output are fed to a Colpitts oscillator-based RF transmitter (i.e., FM transmitter) for wireless blood flow monitoring. The FM transmitter was designed to carry a base frequency of $81 \mathrm{MHz}$.

Such ultrasonic power-in, RF data-out interrogation scheme can be demonstrated in the stent design and the fabrication process is shown in Figure 2. The custom pattern and the cantilever-type sensor are machined out of PVDF using a cutter/plotter, Figure 2(a)(b). After completing the electrical connections, a thin layer of polydimethylsiloxane (PDMS) is deposited for passivation, Figure 2(c). During curing (after $30 \mathrm{~min}$ ), the sample is removed and placed in a vacuum oven for full curing, Figure 2(d). This process introduces a very thin layer of passivation $(\sim 10 \mu \mathrm{m})$. Then it is rolled into a cylindrical shape and sutured using FDA-approved nylon thread. The active stent is completed by assembling electronic components on the surface, Figure 2(e). Figure 2(f)-(g) shows pictures of a prototype. The overall stent dimension was $30 \mathrm{~mm} \times 10 \mathrm{~mm}$ (thickness $=110 \mu \mathrm{m})$, and the flow sensor was $7.5 \mathrm{~mm} \times 4 \mathrm{~mm}$.

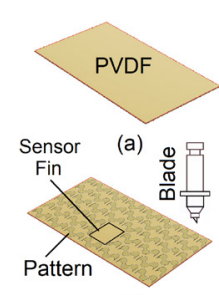

(b)

(c)

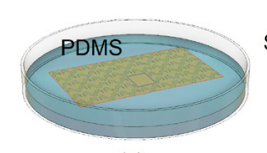

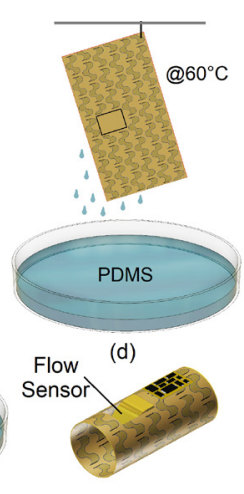

(e)

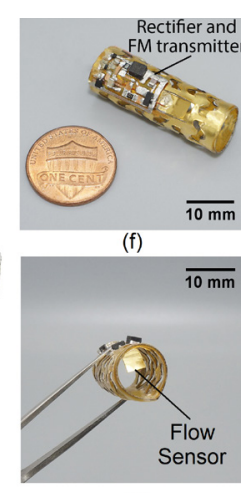

(g)
Figure 2: Fabrication process: (a) planar PVDF; (b) patterning with cutter/plotter; (c) submerging into PDMS for passivation; (d) half-cured PDMS dripping to form a thin layer; (e) rolled and sutured; (f) prototype; (g) top view shows the flow sensor fin.

The prototype of the active stent was characterized in vitro using the experimental setup illustrated in Figure 3. A sinusoidal 
signal from a function generator is fed into an RF amplifier to drive an ultrasonic transducer (Lead zirconate titanate, PZT5H, Piezo Inc.) to operate at $6.7 \mathrm{MHz}$ frequency while its acoustic intensity was below FDA limit $\left(720 \mathrm{~mW} / \mathrm{cm}^{2}\right)$. A mass flow pump modulated the water flow to mimic a blood flow through closed-loop tubing while its flow rate was monitored by an inline digital flow meter and an active stent flow sensor. The sensor readout was monitored by a spectrum analyzer which was placed approximately $1 \mathrm{~m}$ away to monitor frequency changes corresponding to the change in flow rate.

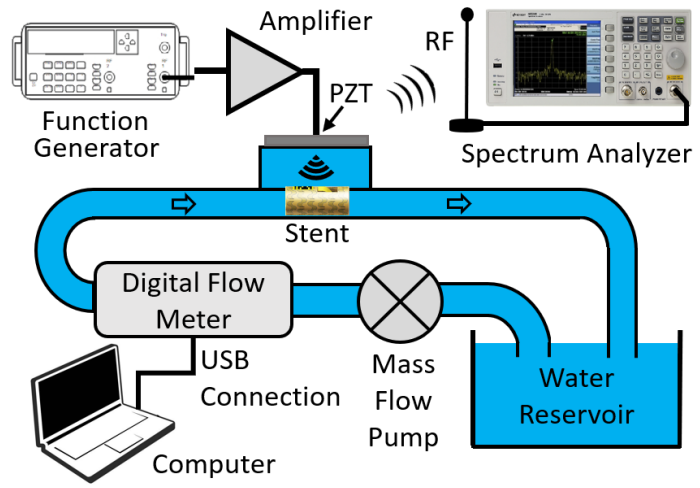

Figure 3: Experiment setup: Amplified sine wave is fed to a PZT transducer to induce ultrasonic wave; mass flow pump mimics blood flow inside tubing; spectrum analyzer is receiving RF output signal from active stent; digital flow meter is used to verify output data.

\section{RESULTS}

The mechanical characterization of the stent designs was performed at first, shown in Figure 4. Figures show the change in stent diameter, Figure 4(a), and change in the angle of the fins (patterns stood up), Figure 4(b), while changing the length of the stent for different samples with various pattern placements varied in arrowed direction, Figure 4(c). As shown in Figure 4, the optimized mechanical performance was $2.4 \mathrm{~mm}$ expansion in diameter and $34.8^{\circ}$ rising in fin angle when the length expansion was $2.91 \mathrm{~mm}$.

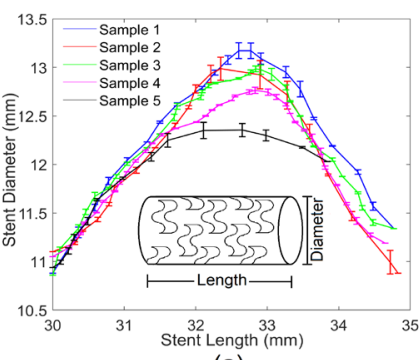

(a)

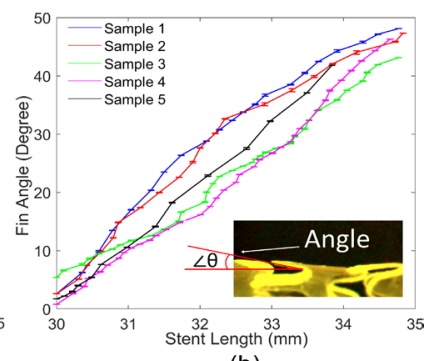

(b)

Figure 4: Mechanical relationship of expansion: (a) diameter changes and, (b) fin angle changes with stent length expansion; (c) Pattern placements varied towards arrowed direction for different samples.

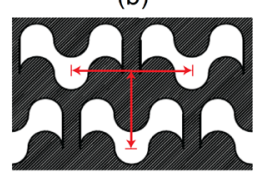

(c)

After the stent platform characterization, the flow sensor and the RF transmitter were characterized. Figure 5 shows the output characteristics of the cantilever-type flow rate sensor. The sensor could measure flow rate ranging from 30 to $980 \mathrm{~mL} / \mathrm{min}$ with a linear sensitivity of $0.16 \mathrm{mV} / \mathrm{mL} / \mathrm{min}$ in $30-200 \mathrm{~mL} / \mathrm{min}$ range and $0.06 \mathrm{mV} / \mathrm{mL} / \mathrm{min}$ in $201-980 \mathrm{~mL} / \mathrm{min}$ range. Wireless sensing readout is shown in Figure 6. The range of the received frequency peak was in $81-88 \mathrm{MHz}$ range on the spectrum analyzer while the flow sensor's flow rate varied between 30 to $980 \mathrm{~mL} / \mathrm{min}$ range.

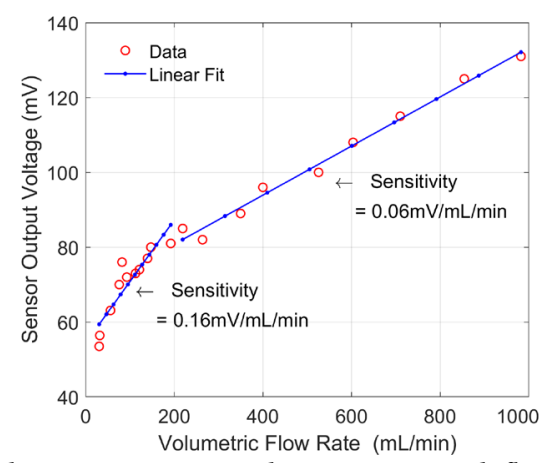

Figure 5: Flow sensor output voltage varying with flow rates.

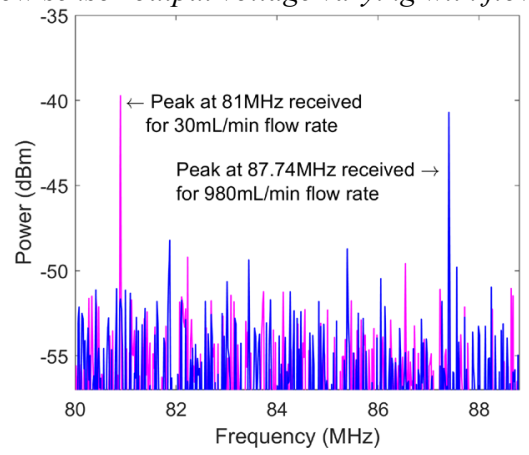

Figure 6: Received frequency on spectrum analyzer for minimum $(30 \mathrm{~mL} / \mathrm{min})$ and maximum $(980 \mathrm{~mL} / \mathrm{min})$ flow rates.

\section{CONCLUSION}

Ultrasonically powered wireless interrogation scheme was developed and implemented in an active stent with a flow rate sensor and an RF transmitter. It is expected to reduce the post-EVAR complication and provide convenient access to diagnostic information of the patients.

\section{ACKNOWLEDGEMENTS}

The authors would like to thank Dr. Dennis Silage for his valuable advice and help with the experimental setup.

\section{REFERENCES}

[1] J. C. Parodi, J. C. Palmaz, and H. D. Barone, "Transfemoral Intraluminal Graft Implantation for Abdominal Aortic Aneurysms," Ann. Vasc. Surg., vol.5, no.6, pp.491-499, 1991.

[2] Greenhalgh, R. (2004). Comparison of endovascular aneurysm repair with open repair in patients with abdominal aortic aneurysm (EVAR trial 1), 30-day operative mortality results: randomized controlled trial. The Lancet, 364(9437), 843-848.

[3] G. Maleux, M. Koolen, and S. Heye, "Complications after Endovascular Aneurysm Repair," Semin. Intervent. Radiol., vol. 26, no. 1, pp. 3-9, 2009.

[4] A. Putra, Y. M. Cheah, N. Muhammad, A. Rivai, and C. M. Wai, "The Effect of Perforation on the Dynamics of a Flexible Panel," Advances in Acoustics and Vibration, vol. 2014, pp. 117, 2014.

[5] Y. Xin et al., "The use of polyvinylidene fluoride (PVDF) films as sensors for vibration measurement: A brief review," Ferroelectrics, vol. 502, no. 1, pp. 28-42, 2016.

[6] B. A. J. Angelsen and A. O. Brubakk, "Transcutaneous measurement of blood flow velocity in the human aorta," Cardiovasc. Res., vol. 10, no. 3, pp. 368-379, 1976.

\section{CONTACT}

*Prof. Albert Kim, albertkim@temple.edu 